\title{
2D Digital Image Correlation in strain analysis by Edge Detection And its Optimisation
}

\author{
P. S. S. Sashank, Kondabolu Nishanth \\ Dept. of ECE Gitam University Vishakhapatnam, India \\ Dept. of ECE Gitam University Vishakhapatnam, India
}

\begin{abstract}
Optical measurements of macroscopic parameters such as strain and displacement have evolved into a special branch of engineering called as stress analysis. Topics such as holography, speckle interferometry, speckle photography have advanced from basic stage of research to their advanced stages and are employed as solution's to many engineering problems, however these systems suffer from stability requirements and consumes much processing time. More recently complete automated procedure for strain analysis has been proposed for computation of strain analysis are been introduced one of the most efficient technique in optical strain analysis is digital image correlation technique. This work includes implementation of two dimensional digital image correlation to aluminum plates from universal testing machine and improvise the technique by the process of edge detection

Index Terms: Digital image correlation(DIC), edge detection, strain analysis, Universal Testing Machine(UTM)
\end{abstract}

\section{INTRODUCTION}

As a practical and effective tool for quantitative deformation measurement of a planar object surface, two-dimensional digital image correlation (2D DIC) is now widely accepted, and commonly used in the field of experimental mechanics directly provides full-field displacements to sub-pixel accuracy and full-field strains by comparing the digital images of a test object surface acquired before and after deformation[1]. In this review, methodologies of the2D DIC technique for displacement field measurement and strain field estimation are systematically discussed[2].

In surface deformation measurement of materials and structures subjected to various loadings (e.g. mechanical loading or thermal loading)the vital considerations are tasks of experimenting solid mechanics and interferometric techniques. Interferometric metrologies require a coherent light source, and the measurements are normally conducted in a vibration isolated optical platform in the laboratory. Interferometric techniques measure the deformation by recording the phase difference of the scattered light wave from the test object surface before and after deformation[7]. The measurement results are often presented in the form of fringe patterns; thus, further fringe processing and phase analysis techniques are required. Non-interferometric techniques determine the surface deformation by comparing the gray intensity changes of the object surface before and after deformation, and generally have less stringent requirements under experimental conditions.

As a representative non-interferometric optical technique, the DIC method has been widely accepted and commonly used as a powerful and flexible tool for the surface deformation measurement in the field of experimental solid mechanics. It directly provides full-field displacements and strains by comparing the digital images of the specimen surface in then deformed (or reference) and deformed states respectively. In principle, DIC is an optical metrology based on digital image processing and numerical computing.

During the past few years, the DIC method has been extensively investigated and significantly improved for reducing computation complexity, achieving high accuracy deformation measurement and expanding application range.

For example, the two-dimensional (2D) DIC method using a single fixed camera is limited to in-plane deformation measurement of the planar object surface. To obtain reliable measurements, some requirements on the measuring system must be met. If the test object is of a curved surface, or three-dimensional (3D) deformation occurs after loading, the 2D DIC method is no longer applicable. To overcome this disadvantage of 2D DIC, 3D DIC based on the principle of binocular stereovision was developed. In this paper the deformation analysis is further improvised by calculating the percentage of deformation using different methods of edge detection.

\section{STRAIN ANALYSIS Through UTM}

Strain analysis is a branch of engineering which deals with the amount of bearing capacity of specimen when it is subjected to force or a load. Generally the input for the strain analysis is a complex material and 
maximum and minimum forces that are to be applied and the output data gives the amount of deformation caused by the force applied[6]. Strain analysis can be performed through Universal testing machine is a fast accurate and a simple experimental setup for strain analysis. The output of this system is a load displacement graph of a specimen
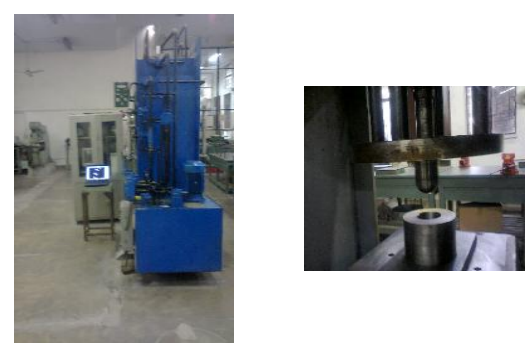

Fig1: Experimental setup of Universal Testing Machine

Basic image acquisition for analysis is taken from a 5megapixel camera with a standard resolution of $4000 \times 2248$ image

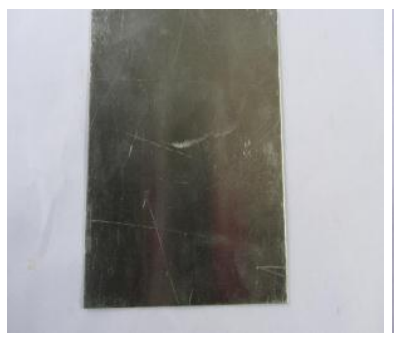

Figure2(a)

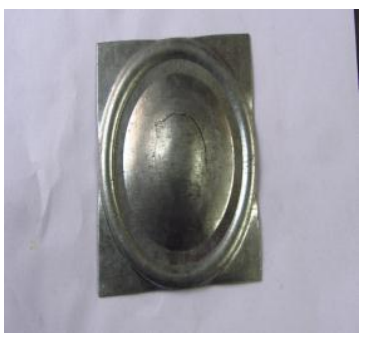

Figure2(b)

(a).Soft aluminum specimen before strain analysis

(b).Soft aluminum specimen after strain analysis

\section{Problem Definition}

The main problem in strain analysis by universal testing machine is to get the accurate percentage of deformation of a specimen. When Digital Image Correlation technique is applied the displacement information can be obtained but the accurate percentage of deformation and the breakage of the specimen cannot be stopped .here is a novel method along with Digital Image Correlation that can get the accurate percentage of deformation but also prevents the specimen to safe at the point of accurate deformation. The approach headed by the implementation of edge detection algorithms to each image captured from the device .

\section{IMPLEMENTATION OF DIC}

\section{A. Displacement field measurement}

Due to the discrete nature of the digital image, the integer displacements with single pixel accuracy can readily be computed. To further improve displacement measurement accuracy, certain sub-pixel registration algorithms should be used. Generally, to achieve sub-pixel accuracy, the implementation of 2D DIC comprises two consecutive steps, namely initial deformation estimation and sub-pixel displacement measurement. In other words, the 2D DIC method normally requires an accurate initial guess of the deformation before achieving subpixel accuracy[4]

In spatial domain, the accurate locations of the target subset can be determined by a fine search routine, pixel by pixel, performed within the specified range in the deformed image. Some schemes, such as the coarseto fine and the nested searching schemes, can be used to speed up the calculation. These search schemes yield one pixel resolution. Alternatively, the correlation between the reference subset and the deformed subset can also be implemented in Fourier domain

Here the 2D DIC is performed on aluminum specimen at the rate of 20 images per second from acquisition device 


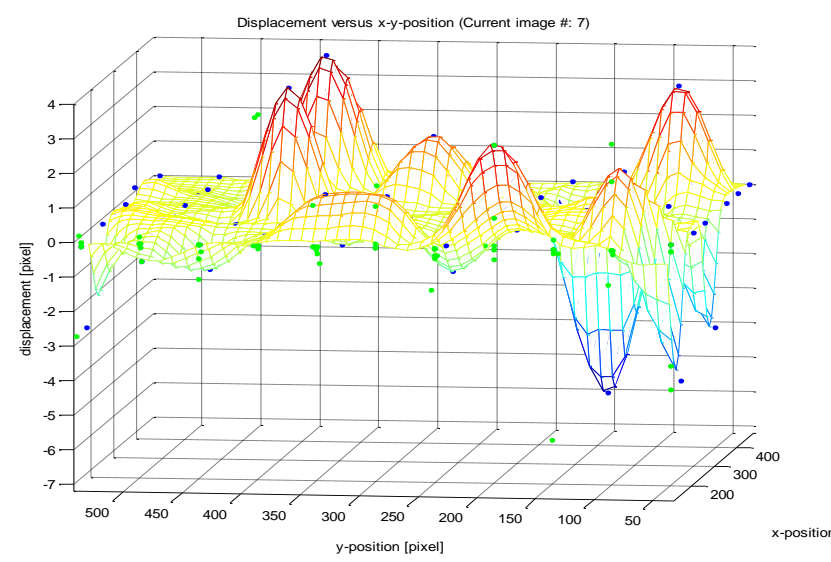

Fig3 : displacement information of 1000 correlated images

\section{B. Strain field estimation}

Now, we can get the full-field displacements to sub-pixel accuracy using the algorithms described above. However, as in many tasks of experimental solid mechanics such as mechanical testing of material and structure stress analysis, full-field strain distributions are more important and desirable. Regrettably, less work has been devoted on the reliable estimation of strain fields. Presumably, this can be attributed to the fact that the displacement gradients (i.e. strains) can be directly calculated using the NR, quasi-NR, LM or genetic algorithm. Alternatively, the strains can be computed as a numerical differentiation process of the estimated displacement[4].
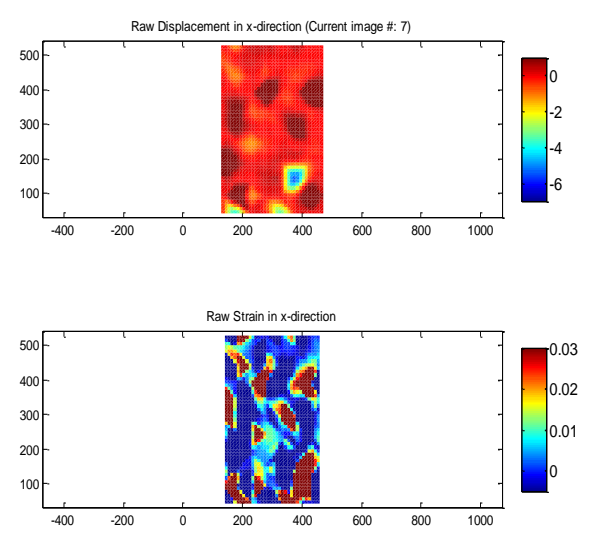

Fig4: strain Field estimations

The results of displacement of pixels to the present position of the pixels and true strain analysis are shown as below

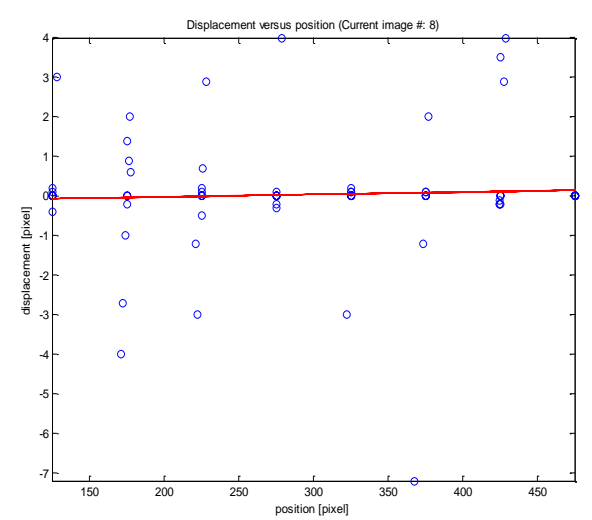

Fig4: displacement of markers to the actual position 


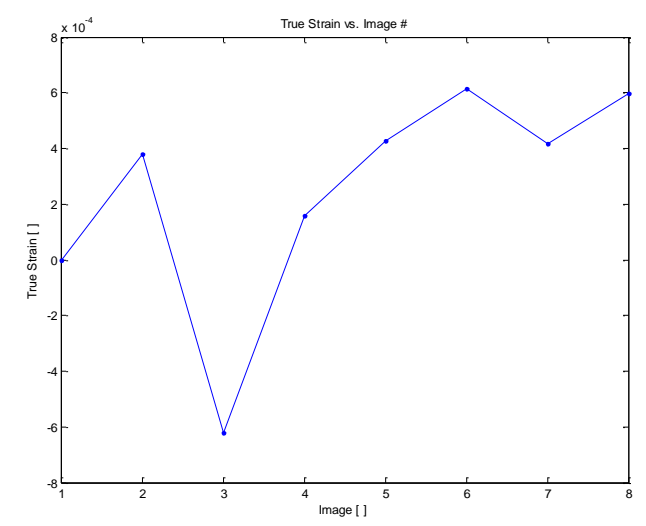

Fig5: True strain representing the deformation

\section{Proposed Algorithm}

The application of the edge detection algorithm in parell with the digital image corellation algorithim gives the accurate percentage of deformation but also stops the excess breakage of the specimen This helps the analysis to stop at a defined point of required deformation

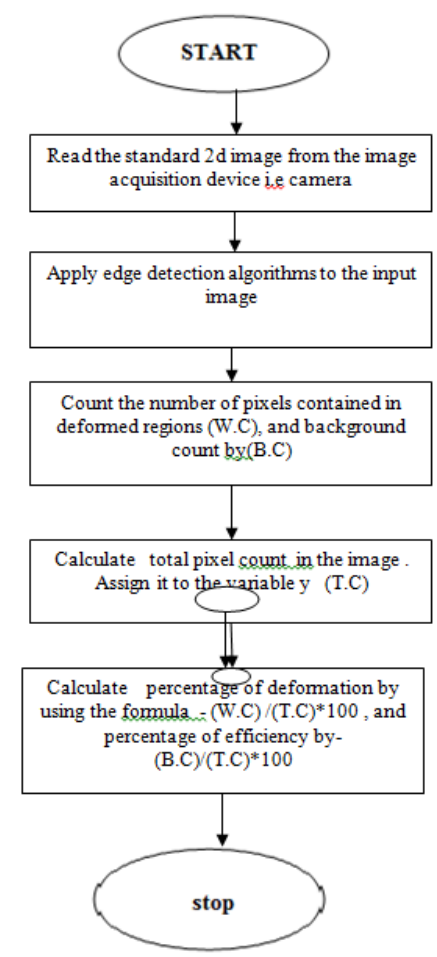

Fig5: Algorithm

\section{EDGE DETECTION}

Edge detection is one of the most commonly used technique in the field of image processing. They characterize boundary information and filters out unnecessary data therefore preserving important structural properties of image. This paper mainly compares various edge detections applied in strain analysis of soft aluminum. Various edge detection techniques include Prewitt, Sobel, Canny, LoG-(Laplacian of Gaussian)and zero cross detectors . Image analysis is performed by using Matlab. The experimental results shows that the Sobel and Robert's operators shown better results when compared to Canny operator for deformation analysis[5].

\section{A. Edge detection techniques}

\subsection{Sobel edge detection}

Sobel operator is called as discrete differentiation operator It performs gradient to image intensity functions. Sobel operator is formed by passing a simple mask over an image. The sobel operator performs a 2D 
spatial gradient measurement over an image and high spatial frequency components are treated as edges so it is computationally very efficient. Here one kernel is simply the other which is rotated by 90degrees

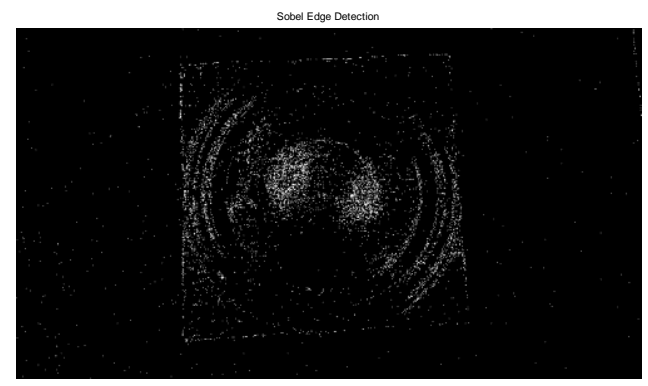

Fig6- sobel edge detection applied to input image

\subsection{Prewitt edge detection}

Prewitt operator is same as sobel operator and is used to find horizontal and vertical edges. The gradient of image intensity varies from darker region to brighter region. This results in more smoothing effect at the edges .It is an efficient way to identify the magnitude of an edge.

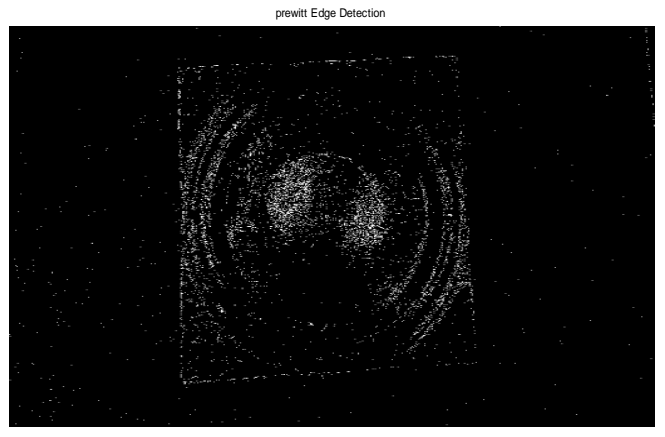

Fig7- Prewitt operator applied to input image

\subsection{Robert's edge detection}

It is the first edge detection algorithm and was introduced by Lawrence Roberts in the year 1963.The differential gradient of the image is obtained by computing the sum of squares of differences between adjacent pixels .It highlights the components of high spatial frequency which corresponds to edges. It is used for simple and quick computation of spatial gradient. Here one mask is simply the other rotated by 90 degrees.

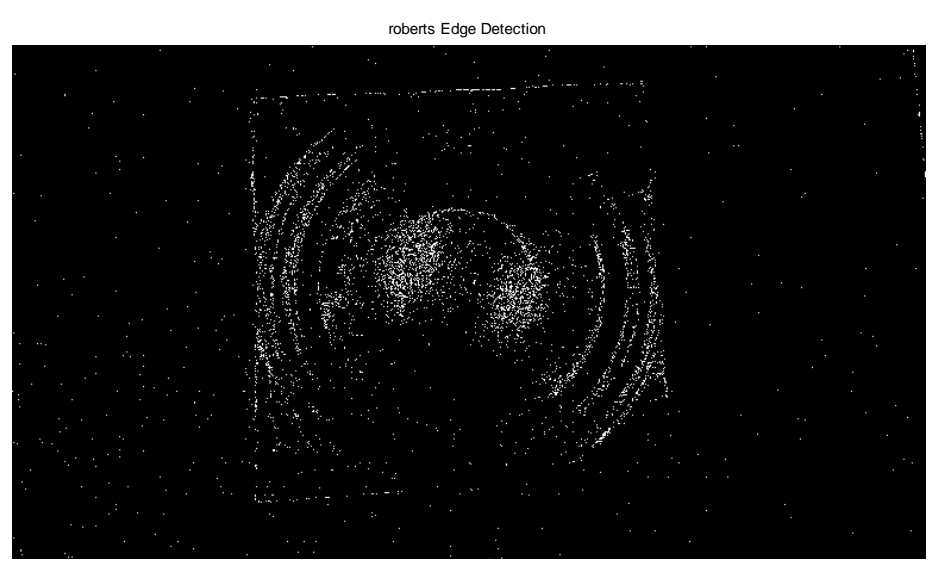

Fig8- Robert's operator applied to input image 


\subsection{Laplacian of Gaussian (LoG)}

LoG is a $2 \mathrm{D}$ isotropic measure of a $2 \mathrm{D}$ spatial derivative of an image. It highlights the rapid intensity changes in an image so it is used for edge detection. It smoothens the image and reduce the noise content of an image. It takes a single grey level as an input image and produces another grey level at output .

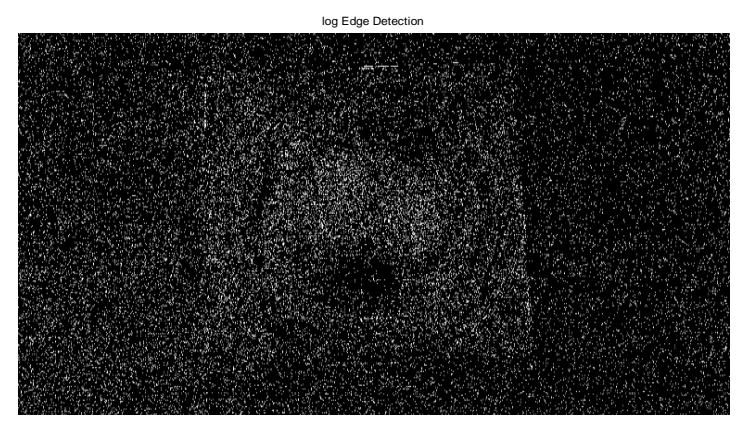

Fig9 - LoG operator applied to input image

\subsection{Zero cross edge detector}

Zero cross edge detector depends on Laplacian sign changes and treat them as edges in images. It is generally mentioned as a future detector rather than edge detector. The output of a zero cross detector is generally a binary image with single pixel thickness showing positions of zero crossing points

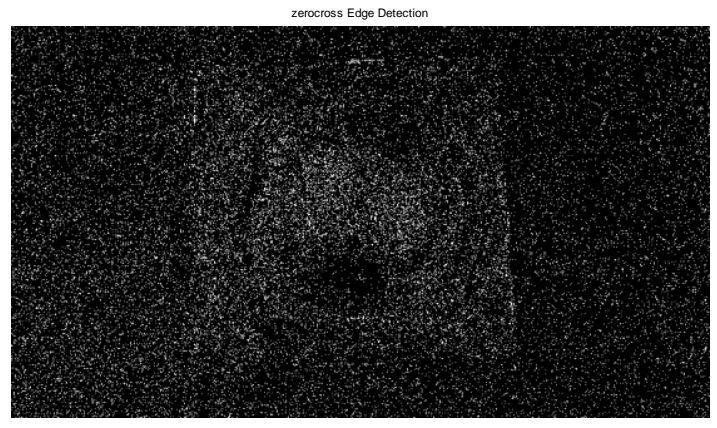

Fig10: Zero cross detector applied to input image

\subsection{Canny edge detector}

Canny edge detector is also called as optimal edge detector. Canny edge detection depends upon gradient magnitude of smoothened image . Local maxima of gradient magnitude high are defined as an edge. The optimal function in canny is described by sum of four exponential terms but can be approximated as first de derivative of Gaussian

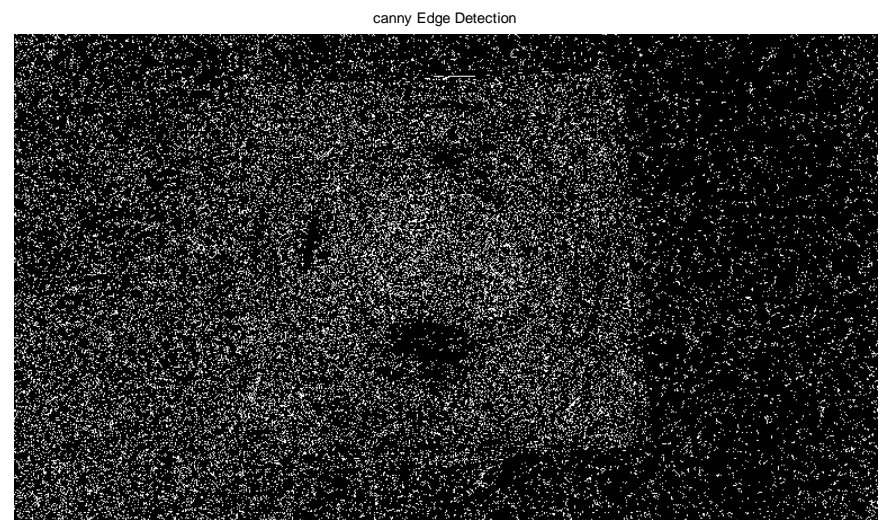

Fig11 - Canny operator applied to input image 
VII. RESULTS

\begin{tabular}{|c|c|c|c|c|c|c|}
\hline $\begin{array}{c}\text { S.n } \\
\text { o }\end{array}$ & $\begin{array}{c}\text { Name of } \\
\text { edge detector }\end{array}$ & $\begin{array}{c}\text { Total } \\
\text { count(in } \\
\text { pixels) } \\
\text { T.C }\end{array}$ & $\begin{array}{c}\text { Black count(in } \\
\text { pixels) } \\
\text { B.C }\end{array}$ & $\begin{array}{c}\text { T.C }- \text { B.C } \\
=\text { White count } \\
\text { W.C }\end{array}$ & $\begin{array}{c}\% \text { of } \\
\text { deformation( } \\
\text { error) } \\
\text { (W.C/ T.C)* } \\
100\end{array}$ & $\begin{array}{c}\% \text { of } \\
\text { efficiency } \\
\text { (B.C/ } \\
\text { T.C)* } 100\end{array}$ \\
\hline 1. & Sobel & 562000 & 479901 & 82099 & 14.6084 & 85.39 \\
\hline 2. & Prewitt & 562000 & 479578 & 82422 & 15.012 & 87.12 \\
\hline 3. & Roberts & 562000 & 504799 & 57201 & 10.1781 & 89.82 \\
\hline 4. & LoG & 562000 & 269125 & 292875 & 52.1130 & 47.88 \\
\hline 5. & $\begin{array}{c}\text { Zero cross } \\
\text { detector }\end{array}$ & 562000 & 292875 & 292875 & 47.8870 & 48.00 \\
\hline 6. & Canny & 562000 & 293460 & 293460 & 48.7829 & 49.79 \\
\hline
\end{tabular}

Fig12: performance analysis of various edge detection algorithms

\section{References}

[1] Roux, Stéphane, Julien Réthoré, and François Hild. "Digital image correlation and fracture: an advanced technique for estimating stress intensity factors of 2D and 3D cracks." Journal of Physics D: Applied Physics 42.21 (2009): 214004.

[2] P. S. S. Sashank, Kondabolu Nishanth/ International Journal of Engineering Research and Applications (IJERA) ISSN: 2248-9622 www.ijera.com Vol. 3, Issue 4, Jul-Aug 2013, pp.2232-2236

[3] Périé, Jean-Noël, et al. "Analysis of a multiaxial test on a C/C composite by using digital image correlation and a damage model." Experimental Mechanics 42.3 (2002): 318-328.

[4] Wang, Y. Q., et al. "Quantitative error assessment in pattern matching: effects of intensity pattern noise, interpolation, strain and image contrast on motion measurements." Strain 45.2 (2009): 160-178.

[5] Canny, John. "A computational approach to edge detection." Pattern Analysis and Machine Intelligence, IEEE Transactions on 6 (1986): 679-698.

[6] Kropatsch, Walter G., and Horst Bischof, eds. Digital image analysis: selected techniques and applications. Springer, 2001.

[7] Bovik, Alan C., Marianna Clark, and Wilson S. Geisler. "Multichannel texture analysis using localized spatial filters." Pattern Analysis and Machine Intelligence, IEEE Transactions on 12.1 (1990): 55-73. 